\title{
A pilot study of optics laboratory activities impact on students connections between theory and experiment
}

Christopher Nakamura, Marie Cassar, Brittany Brady

Christopher M. Nakamura, Marie T. Cassar, Brittany A. Brady, "A pilot study of optics laboratory activities impact on students connections between theory and experiment," Proc. SPIE 11143, Fifteenth Conference on Education and Training in Optics and Photonics: ETOP 2019, 1114339 (2 July 2019); doi: $10.1117 / 12.2523598$ 


\title{
A Pilot Study of Optics Laboratory Activities' Impact on Students Connections Between Theory and Experiment
}

\author{
Christopher M. Nakamura*a, Marie T. Cassar ${ }^{\mathrm{b}}$, and Brittany A. Brady ${ }^{\mathrm{b}}$ \\ ${ }^{a}$ Saginaw Valley State University Physics Dept., 7400 Bay Rd., University Center, MI, 48710; \\ baginaw Valley State University Psychology Dept., 7400 Bay Rd, University Center, MI 48710
}

\begin{abstract}
Research in Physics Education suggests students have difficulties in learning physics content through experimental lab work ${ }^{1}$. In applied areas, such as optics, the formation of a strong understanding that spans both theory and application is often necessary. In this pilot study, the progression of six optics students was tracked over a 15-week semester. We integrated short laboratory activities into a traditional upper-division theoretical optics course with the aim of helping students to make connections between concepts in theoretical optics and their implementation/applications in the laboratory. Three interviews were conducted at the beginning, the middle and the end of the semester to monitor the development of students' skills and knowledge in the relevant optics areas. The interview protocols asked students to make conceptual explanations, perform theoretical calculations and perform experimental tasks to demonstrate their understanding. Initial analysis of the interview responses reveals the gaps in the students' knowledge and skills. We performed a qualitative thematic analysis of the interview data to examine student success in making connections between theory and application. Our results consist of a revised interview protocol for assessing learning in this integrated optics context, revised laboratory activities, and insights for experimental designs that could better assess student learning gains.
\end{abstract}

Keywords: Optics Education, Laboratory Activities, Conceptual Understanding Applied Optics

\section{INTRODUCTION \& BACKGROUND}

Optics is an inherently applied field in which technological tools have historically followed scientific understanding very quickly, and those technologies have been broadly enabling beyond optics, leading to advances in chemistry, biology and engineering. Broadly, the study of optics is at least as motivated by applications as by the inherent value of understanding the physical behavior of light. A practical familiarity with experimental, and applied aspects of optics is often necessary for success in careers in optics. At the same time understanding the theory of optics is important for the design and implementation of new optical technologies. In fact, the interconnection of optics theory and experimental/applied optical implementation is very high and learning outcomes in which students are capable of making and exploiting these connections in effective ways are very desirable. Likely, at least in part, for this reason, and similar to electronics, optics lab courses are often seen in upper-division physics programs, as well as in optics programs, and optical engineering programs. However, there is recent evidence from physics education research (PER) that students struggle to learn theoretical physics concepts from laboratory exercises ${ }^{1,2}$. This literature suggests, however that laboratory courses may still be quite useful for teaching knowledge and skills particularly relevant to experiments ${ }^{1,2}$. Conversely, the idea that students would learn experimental/applied skills from studying theoretical physics is very counter-intuitive. While it is not completely impossible for studies of theoretical physics to lead to improved experimental skills that is not the way we usually think of students learning those skills. Our assertion that optics advances hinge on a well-honed understanding of both theoretical and experimental aspects of the discipline then presents a difficulty, for students must understand how these two aspects are related. Ideally, they would be able to recognize the necessary consequences of optical theory in experimental results, predict how an optical system will behave based on theory, and modify the design of an optical system to produce a desired outcome based on theory. Clearly, this is aspirational for students who are just beginning their study of optics, but that is ultimately the desired learning outcome. The addition of a laboratory component is one traditional way to ensure that both theory and experiment are addressed in the curriculum. However, this has several associated difficulties. First, it requires a

*cnakamur@svsu.edu

Fifteenth Conference on Education and Training in Optics and Photonics: ETOP 2019, edited by

Anne-Sophie Poulin-Girard, Joseph A. Shaw, Proc. of SPIE Vol. 11143, 1114339 - (C) 2019

SPIE, ICO, IEEE, OSA · CCC code: 0277-786X/19/\$18 · doi: 10.1117/12.2523598 
modification to the course catalog and course schedule. Students will have to take another course and an instructor will have to be assigned that course. Second, as mentioned before the utility of the lab for teaching theoretical ideas is called into question. What we really want is to develop integrated understanding.

An important question that must be addressed is: what constitutes a laboratory activity? There are many dimensions that can be varied here, in terms of what students actually do in the lab, and in what context. The most relevant for our purposes are: the depth of investigation, the length of investigation, and the level of autonomy and independence granted/expected. For the purpose of our research we would consider activities, which have a high level of these qualities to be optics projects, which more closely resemble extended research activities. There can be little doubt that such projects can be very productive for student learning, and there is literature that discusses learning in such contexts. However, the demands of such projects are beyond our design constraints, particularly our time constraints, but also our resource constraints. In this work we want to explore the other end of the spectrum. Our activities would best be described as laboratory exercises or laboratory demonstrations. They are necessarily short and targeted at quickly and clearly demonstrating a particular aspect of optical theory using simple equipment that is commonly employed in optics lab (research and/or industrial). In this sense, our activities are designed to begin in a conceptually focused manner: students are tasked with observing an effect and making related quantitative measurements. At the same time, we sought to avoid "cook-book" laboratory exercises, and so although we try to make the goal as clear as possible, we do not set out a step-by-step procedure by which the students are to do it. The procedures need to be figured out. Furthermore, the activities were generally designed to allow for open-ended exploration beyond the required tasks. There is prior history of trying to teach an optics course through experimental methods, albeit with a more phenomenological approach ${ }^{3}$.

In this paper we describe an exploratory pilot study in which we introduced 6 short laboratory activities into a traditional junior/senior level optics course in an attempt to help students develop integrated understanding of both theoretical and experimental aspects of optics without introducing a separate laboratory component. The research design is a qualitative case-study in which student learning was monitored by voluntary interviews over the course of the semester.

\section{RESEARCH CONTEXT: COURSE DESIGN AND LABORATORY ACTIVITIES}

\subsection{Course Description}

Physics 402 at Saginaw Valley State University is a traditional 3 credit hour junior/senior level optics course targeted primarily at physics majors, although some engineering students take it as an elective. The course typically has an equal weighting of geometric optics and wave optics. Enrollment is typically between 5 and 10 students. The textbook for the course varies between instructors, but is typically a standard choice such as Optics by E. Hecht or Introduction to Optics by F. Pedrotti and L. Pedrotti. In the semester of this study the latter text was used. The course met two days a week, and each class was 80 minutes in duration. Traditionally there has been no laboratory component to the course. The curriculum includes a stand-alone laboratory course on "Modern Optics and Holography" which shares some content overlap with Physics 402, but generally focuses on more-advanced experimental techniques.

\subsection{Laboratory Activities}

Six laboratory activities were distributed over the course of the 15 -week semester. The laboratory activities were designed with several pedagogical goals in mind and with several constraints imposed. Pedagogically the first goal was to demonstrate important aspects of the theory being discussed in the course so as to clarify and reinforce the theoretical concepts. The second goal was to develop experience and competence working with common equipment used in research optics labs both in academia and industry. Ultimately our goal is to achieve these goals in an interdependent manner such that the understanding of the theory informs what students do with the equipment and their experiences with the equipment support the development of their understanding of optical phenomena. Our course structure constrained the amount of time available for each lab to 80 minutes. We did not view this as a major constraint because we wanted the laboratory activities to be primarily demonstrative, especially at first. The core of each activity was designed to be completed in a little over an hour and additional exploratory prompts were provided for students to go further. We hoped that students would want to engage in more exploratory investigations based on the work they had done in lab and additional open lab time was provided to further explore the relevant phenomena. Students generally completed the activities in pairs, but a group of three was often necessitated by the odd number of enrolled students. Students were held accountable for the lab work in the form of keeping a laboratory notebook, which counted for $17 \%$ of their course grade. 
1. Beamline Alignment Lab - In this lab students need to learn how to align a laser beam to two irises using kinematic mirror mounts. Emphasis is on building some familiarity with normal optics lab equipment, and also understanding how the equipment works. Questions about how much angular displacement a linear actuator with a certain number of threads per unit length can produce, as well as the sensitivity of the mount actuators are addressed.

2. Radiometry/Photometry Lab - In this lab students explore the difference between radiometric measurements and photometric measurements. A demonstration using red and green LEDs highlights the importance of the eye's relative sensitivity at different wavelengths by illustrating that when the red and green LEDs appear equally bright the red LED emits significantly more light than the green one (as measured by a wavelength calibrated photodiode detector), in accordance with the photopic response of the eye.

3. Aberrations Lab - In this lab students look at, and make measurements with, optical setups designed to highlight spherical aberrations, chromatic aberrations, coma, and astigmatism. Emphasis is on identifying the features, which characterize each aberration.

4. Telescope \& Microscope Lab - In this lab students make simple two lens refracting telescopes and make measurements/observations designed to illustrate theoretical calculations performed in class and on homework.

5. Polarization Optics Lab - In this lab students demonstrate Malus' law, among other physical aspects of polarization using polarizers and a photodiode detector. The use of a quarter-wave plate as an optical isolator is demonstrated.

6. Interference \& Diffraction - In this lab students make quantitative measurements of various diffraction patterns produced by familiar configurations (single slit, two slit) and less familiar configurations (half barrier, for example).

Two additional lab activities, one exploring dispersion in a prism and a transmission grating, and one exploring reflection and transmission coefficients for a laser incident upon an air-glass boundary were also developed but not implemented primarily due to time constraints in the course. We intend to implement these in the next course offering.

\section{RESEARCH DESIGN AND METHODOLOGY}

The small number of enrolled students led us to implement a qualitative, case-study research design, as described below. Although we understood this would limit the degree to which we could legitimately generalize our findings, we believed that sufficient insight could be found from studying the effect of the activities in this qualitative manner to continue their development and progress towards larger, collaborative studies that have greater capacity to generalize.

\subsection{Interview Design}

To study the effects of our laboratory activities, we use a case-study design in which participating students were interviewed 3 times over the course of the semester as a means of assessing the development of their knowledge of relevant optics ideas. Each interview followed the same format. Students were presented with a series of 12 tasks arranged into four groupings of 3 questions. The four groupings brought together questions focusing on theoretical geometric optics, experimental geometric optics, theoretical wave optics and experimental wave optics. Experimental tasks typically presented students with some equipment and asked them to demonstrate an effect or achieve an experimental outcome and then explain how/why it worked. Theoretical tasks required students to perform a calculation and explain the ideas behind it, or to explain the theoretical ideas behind a concept. An example question prompt that focuses on a conceptual explanation is shown in Figure 1.

An important ethical consideration associated with this study is the potential for pressure from the instructor to participate or fear of retribution for not participating. Students must feel free choose whether or not participate in a study. Even in the absence of malicious intent, subconscious effects might result in negative consequences for students opting not to participate. To that end, one of us (Cassar) recruited participants in the course and conducted the interviews. Another (Nakamura) taught the course, and throughout the semester was unaware of which students were participating and which were not. Of seven students enrolled in the course, six chose to participate in the study and five completed all three interviews. The research design was reviewed and approved by the SVSU Institutional Review Board (IRB) to ensure compliance with ethical research practices.

Interviews were conducted at the beginning, middle and end of the semester and were planned to take about an hour and in practice students completed the protocol in about 60-80 minutes. Interviews were audio and video recorded 
with informed consent from the participants. Data was also collected in the form of written artifacts generated by the students as they responded to the question prompts. Students could ask the interviewer for information that they needed but could not remember, but the interviewer (by design) was limited in what information she could provide. Students who needed an equation that they could not remember would be provided that information. However, generally it was expected that they would provide the conceptual understanding, mathematical reasoning and experimental familiarity by themselves. The interviewer being a person whose expertise was outside of physics undoubtedly influenced the manner in which students answered the question. We believe the question prompts and course context made it clear that a rigorous physical explanation was necessary, while the interviewer made it clear that the explanation should also be broadly comprehensible.

\section{Task 4-a}

In the image below a laser beam is being shined on a two-slit assembly. A screen is located a significant distance away. A set of interference fringes becomes visible on the screen, as indicated. What is the phase difference between the light from slit 1 and the light from slit 2 at point $P$. How do you know?

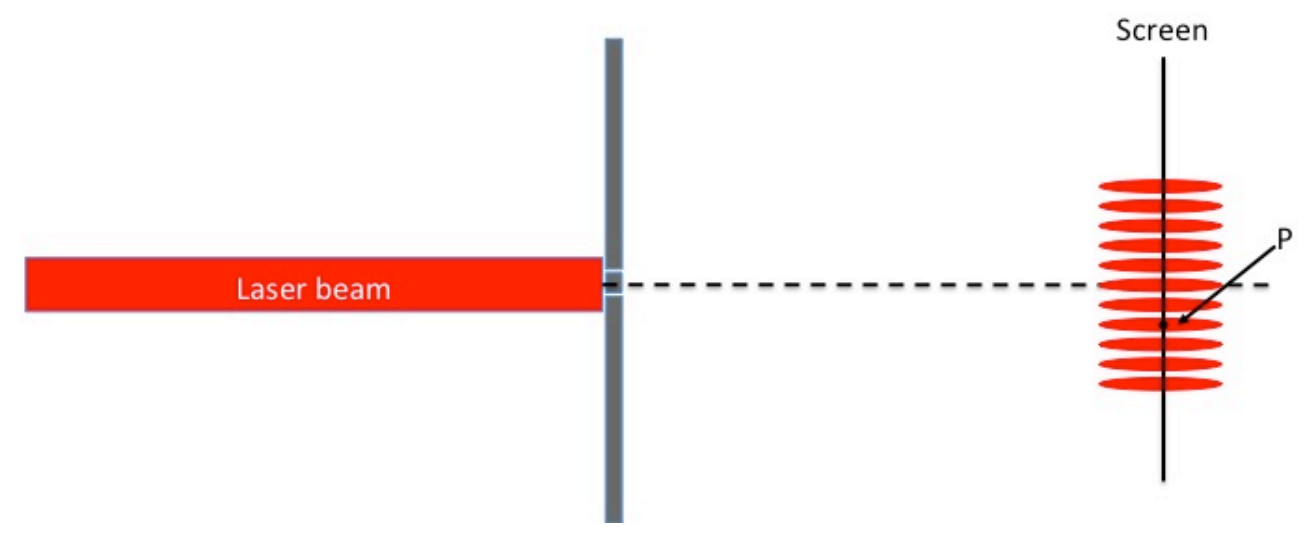

Figure 1. An example question prompt for our interview protocol. Students are presented with an optical context in which they must demonstrate understanding of the phenomenon. Questions typically have an objective right/wrong component (in this case, the phase difference) and an open-ended component in which they explain their understanding. Half the questions require that they demonstrate/explain using optical components and half required just a theoretical/conceptual explanation that did not involve equipment.

\section{INTERVIEW DATA ANALYSIS}

\subsection{Interview Data Analysis Scheme}

While it is sometimes obvious a priori what the analysis scheme should be for a qualitative research study, frequently it is not. In such a case the analysis scheme should be constructed based on what is seen in the data ${ }^{4}$. In this study two of us (Cassar, Brady) have carefully reviewed student responses to a subset of the data in order to produce a general coding scheme that is useful for understanding the strengths and deficiencies of a student's response to a particular interview question and which could be used to this end in analyzing a wide range of interview questions. We proceeded from the recognition that ultimately what we want to do is to describe the process by which students productively or unproductively construct answers to the questions. The analysis scheme is shown in Figure 2.

The Interview Data Analysis Scheme created for coding the students' answers contains six main sections. The sections of the analysis scheme represent the high level, generic stages in reasoning observed across the students' answers, keeping in mind that any one interview question may or may not have required every stage in the coding scheme to arrive at a correct answer. These sections aim to record the features of the students' reasoning process while 
generating his or her answers to the questions. The analysis scheme was also influenced by the framework proposed by Evans' heuristic-analytic theory of reasoning ${ }^{5}$. The sections in the scheme are the following: Question Analysis, Student Provided (information), Errors, Solution Process, Systematic Analytical Process and Conclusion.

The Question Analysis section codes the extent to which a student mentioned the given factors provided in the question. The Student Provided section then codes additional information (i.e., a mathematical formula, informal knowledge, and/or formal knowledge) the student introduced in the process of generating an answer. Instances in which students inserted irrelevant factors or confused information is coded in the third section, Errors. The fourth section, Solution Process, codes the student's use of verbal reasoning, drawing, computation, and/or equipment while generating an answer. Whether or not the student reviewed and/or overtly tested his or her reasoning is coded in the fifth section, Systematic Analytical Process. Finally, the sixth section codes the degree of accuracy and completeness in the student's answer.

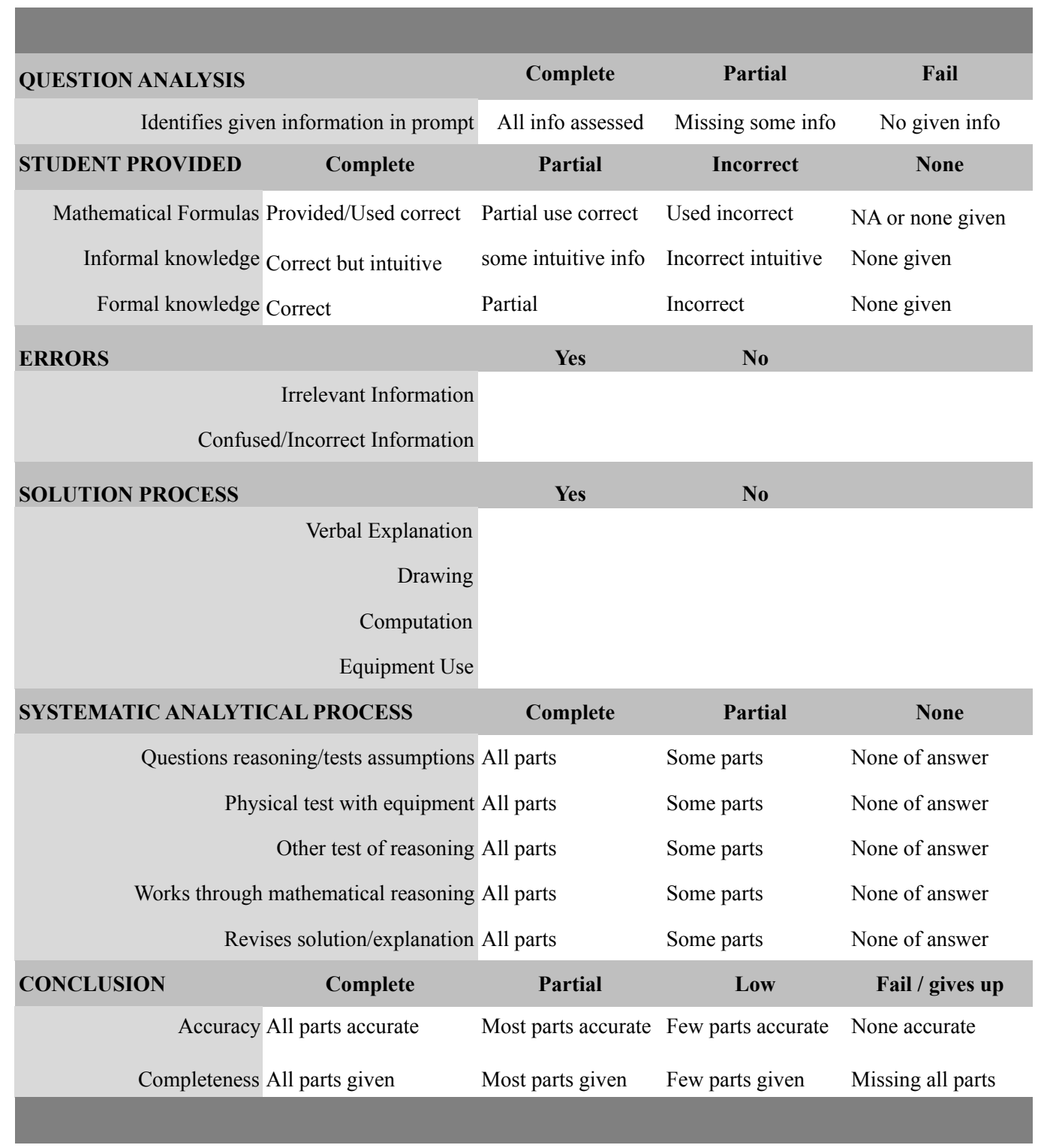

Figure 2. Our qualitative analysis scheme for analyzing student responses to interview question prompts. 


\section{INSTRUCTOR OBSERVATIONS}

One of the authors (Nakamura) was the instructor for the course and observed the students while they completed the laboratory activities as well as their interactions and performance in the rest of the course. While these observations do not rise to the level of data from which generalizable inferences should be extracted, they can provide insight for the revision of the laboratory activities and future research designs that test their efficacy. Most of these observations will not come as a surprise to instructors, as they show general aspects of student behavior that find quite common in other courses as well, but we believe there is still value in explicating them to aid the design process. The instructor's observations are largely related to students' lack of experience (which is expected) and the time necessary to build that experience. The instructor observed that students often seemed reluctant (or unable) to follow systematic procedures in their investigations. Varying of parameters and adjusting of optical systems was often done in somewhat erratic manner, with a low level of control. This is not surprising, as the students had little or no experience with the optics experiments they were doing, or optics experiments in general. At the same time, they did have prior experience doing physics labs, but that seemed to be of little help to them in inferring how to proceed in these lab activities. Advice from the instructor on this front was of limited help. Students seemed to struggle to develop or recall theoretical models of the lab equipment, with discussions with lab partners, or between lab groups providing some help. In general, it took a long time for students to become comfortable with the ideas and with the equipment. Students demonstrated enthusiasm to explore, but also a desire to quickly get the result they were looking for and complete their tasks. Open lab time was very underutilized. The general observation of the instructor is that students would need a lot more time and experience working in the lab to build the knowledge and skills we aim to develop. Students will likely not have time, or be willing to prioritize additional time in the lab if it is not required or strongly incentivized in some other way. We are exploring ways to address these issues and improve both our activities, and they way they are implemented in the course to allow and encourage students to spend more time exploring the optical phenomena, develop more formal exploration strategies, and better connect to the theoretical ideas.

\section{DISCUSSION}

\subsection{Revisions to the Laboratory Activities}

Our analysis of interview data is ongoing, and so our revisions are based on the preliminary observations we have made thus far and an emerging picture of how to best do experimental work from the PER literature. PER has suggested that students engaging in more research-like investigations may be better for the development of reasoning and scientific skills than engaging in lab exercise-like investigations ${ }^{6}$. Additionally, students' feelings of ownership of their work looks to be an important consideration? ${ }^{7}$. These considerations suggest activities that are both more openended and more student-driven should be considered. These modifications present difficulties. In particular, it is consistent with the instructor's observations that students need more time to explore without a goal in mind, but they are likely not well-prepared to do that in an efficient manner. Additional laboratory time must likely be made available for students to struggle and to explore as they develop their skills. Simply telling them how to do things better will likely not help. Establishing connections between activities to make the experience more like a research environment is one possibility to consider, with the goal of providing them a more natural context for developing ownership of their work based on confidence and competence developed in the lab over time. We are also exploring the possibility of a final report, or presentation that places higher emphasis on summative learning than simply grading a lab notebook can. In this way we can incentivize revisiting prior experiments to see what could be learned from them with the benefit of more experience. This additional lab time will likely come at the expense of other lecture time (or time outside of class on the students' parts), and so we are assessing what lecture topics and homework problems can be omitted or reduced in emphasis. For example, geometric derivations, for example take a lot of time, but may not be retained well by students or of highest value for their future efforts.

\subsection{Revisions to the Interview Protocol}

Our primary observation is that our present interview protocol is too long. We are working to develop a shorter protocol, perhaps 8 question prompts that still connect concepts across interviews to understand how students are learning throughout the optics course. Anecdotally, we observed a qualitative difference in how some students have answered some questions, a distinction we might characterize as talking about the answer to the question versus answering the question. In both cases some knowledge of the relevant topic is conveyed and some information about how much is understood can be gained, but we note that the strategy of talking about the answer can be employed to 
keep the discussion on a more superficial level that masks what is known and what is not. This behavior is understandable when a student feels they should know something and does not. We do not want to stigmatize not knowing, in part because we need to gauge that information to assess our materials and in part because it is natural for students to not know some of the material they are just now learning. We are exploring ways to prompt students to become increasingly precise and detailed in their responses so that we can see how and where their understanding of the phenomenon breaks down without discouraging them from open, honest discussion.

Ultimately, we would like to test these lessons in an experimental design. We are working to develop a protocol in which questions are more tightly tuned to the course content as it is discussed in the course. In this way we can more precisely ask questions that precede, succeed, or align with material presently being discussed to better gauge whether students are learning the material and how.

\section{CONCLUSIONS \& FUTURE WORK}

We have implemented a series of laboratory activities in the context of an upper-division optics course in an attempt to help students build connections between their understanding of theoretical concepts in optics and their practical ability to recognize and apply those ideas in a laboratory setting. We have used a qualitative case-study approach to studying the activities and have discussed our progress in developing a scheme for analyzing data from such a design. While there is evidence from PER that establishing such knowledge from laboratory activities is difficult, the importance of those connections in optics suggests this is a useful area to continue researching. Our plan is to continue exploring the use of these, and other related laboratory activities and to study their effects. The physical optics course at SVSU will be offered in Winter of 2020 and it is our intention to again structure the course to include approximately 6-8 laboratory exercises, refined based on the ideas discussed in section 6.1, and to conduct an interview-based research design to again observe student reasoning and learning over the course of the semester.

\section{ACKNOWLEDGEMENTS}

We acknowledge financial support from the Michigan Space Grant Consortium through a Research Seed Grant, and Saginaw Valley State University through an Undergraduate Research Program (UGRP) grant. We acknowledge the contributions of Morgan L. Bucko and Nolan Taylor to the laboratory activities. Nakamura acknowledges productive discussions with Dimitri Dounas-Frazer.

\section{REFERENCES}

[1] Holmes, N.G., Olsen, J., and Wieman, C.E. "Value added or misattributed? A multi-institution study on the educational benefit of labs for reinforcing physics content," Phys. Rev. Phys. Educ. Res. 13(1), 010129, (2017).

[2] Holmes, N.G., Wieman, C.E. "Measuring the impact of an instructional laboratory on the learning of introductory physics," Am. J. Phys. 83(11), 972-978, (2015).

[3] Sorensen, C. M., McBride, D. L., and Rebello, N. S., "Studio optics: Adapting interactive engagement pedagogy to upper-division physics," Am. J. Phys. 79(3), 320-325, (2011).

[4] Cresswell, J. W., [Qualitative Inquiry and Research Design Choosing Among Five Approaches], Sage Publications, Thousand Oaks, 73-75, (2007).

[5] Evans, J. ST. B. T., "The heuristic-analytic theory of reasoning: Extension and evaluation," Psychon. Bul. \& Rev., 13(3), 378-395, (2006).

[6] Holmes, N. G., Wieman, C. E., \& Bonn, D. A., "Teaching critical thinking," Proc. Nat. Acad. Sci., 112(36), 1119911204, (2015).

[7] Dounas-Frazer, D. R., Stanley, J. T., and Lewandowski, H. J. "Student ownership of projects in an upper-division optics laboratory course: A multiple case study of successful experiences." Phys. Rev. Phys. Educ. Res. 13(2), 020136, (2017). 